\title{
A Genetic based Neuro-Fuzzy Controller System
}

\author{
Mohamed, A. H. \\ Solid State Dept., National Centre for Radiation Research \\ and Technology (NCRRT), Cairo, Egypt.
}

\begin{abstract}
Recently, the mobile robots have great importance in the manufacturing processes. They are widely used for assembling processes, handling the dangerous components, moving the weighted things, etc. Designing the controller of the mobile robot is a very complex task. Many simple control systems used the neuro-fuzzy controller in the mobile robots. But, they faced with great complexity when moving in unstructured and dynamic environments. The proposed system introduces the uses of the genetic algorithm for optimizing the parameters of the neuro-fuzzy controller. So, the proposed system can improve the performance of the mobile robots. It has applied for a mobile robot used for moving the dangerous and critical materials in unstructured environment. Its results are compared with other traditional controller systems. The suggested system has proved its success for the real-time applications.
\end{abstract}

\section{Keywords}

Fuzzy controller, Neuro-fuzzy Controller, Optimization, Modeling, Genetic algorithm.

\section{INTRODUCTION}

One of the main problems faces the autonomous mobile robots is their moving in unstructured and dynamic environments. The success of any mobile robot for moving inside these environments based on its ability to have the needed information about the environment and plan for the suitable path that can avoid any obstacle.

Many techniques are used to solve the navigation problems of robot for manage the avoiding of both static and dynamic obstacles. The common techniques such as potential field analytical and the graph-based techniques have been used to overcome these problems. But, all such methods may not be suitable for on-line implementations due to their computational complexities and limitations.

Recently, researches introduced the use of various artificial intelligent techniques such as fuzzy logic, neural network and genetic algorithm to solve these problems [1].

The present research introduces a new methodology that can optimize neuro-fuzzy controller system. It integrates the fuzzy logic, neural network and the genetic algorithm to optimize the parameters for the mobile robot to overcome its navigation problem.

The reminder of this paper is organized as: Section 2 describes the neuro-fuzzy systems. Section 3 represents an overview about the control system of the mobile robots. While, section 4 introduces an overview of the genetic algorithms and their integration with the fuzzy - neural network systems. Section 5 deals with the proposed genetic algorithm Fuzzy-Neuro Controller and its applicability for the mobile robot. Section 6 represents the conclusion.

\section{NEURO-FUZZY SYSTEM}

Neuro-Fuzzy systems, as hybrid systems, have become popular in the last decade. They are combinations of the theories of fuzzy logic and neural networks [2]. In these systems, the parameters of the fuzzy system are determined by means of the learning algorithms used in neural networks. This means that the "black box" nature of neural networks is avoided [3]. So, the fuzzy neural systems are actually used to create or enhance neural networks [4]. Neuro-Fuzzy Systems have proved their success in the classification, diagnosis, pattern recognition, etc. But, they still have some limitation in solving design, planning and predication problems.

Therefore, for these limited types of problems, researchers interested in neuro-fuzzy hybrid systems focused on how to generate an optimal number of neurons or fuzzy rules in a neuro-fuzzy system and investigate automated methods of adding and pruning neurons. A novel strategy used to generate a compact fuzzy neural network [5-7].

\section{CONTROL OF MOBILE ROBOTS}

A mobile robot is a situated and embodied agent endowed with mobility; and operates autonomously, communicating with, and exploring its environment. Mobile robots are complex systems moving in real environments and their controller is designed to control their motion. This complexity appeared due to the need for realizing multiple behaviours respond to multiple sensors are successfully achieved by using the task based decomposition of robot architecture. However, researchers have used the behaviour-based control instead of the conventional robot control due to its great complexity in modeling the real world [8]. Intelligent strategies like fuzzy logic and neural network have been used to endow robot with intelligent capability to navigate its environment autonomously. But, in some applications, the robot can face with a problem to move in unknown or unstructured environments. So, designing the control of such mobile robots need much higher level of intelligence that enables it to adapt successfully its path when environment is changed [9]. These open designing the control problems increase the need for intelligently optimizing the adaptation plan of the controller of the mobile robot to deal with its dynamic environment. The proposed system can use the genetic algorithm to optimize the parameters of the fuzzy-neuro control system to overcome the navigation problem of the mobile robot.

\section{GENETIC ALGORITHMS AND THEIR INTEGRATION BETWEEN FUZZY AND NEURAL NETWORK SYSTEMS}

The Genetic Algorithm (GA) Genetic algorithm (GA) is a global, parallel, stochastic search methods, founded on Darwinian evolutionary principles. Genetic algorithms are suitable for optimizing discrete variables and non-continuous cost functions. They use of the principles of gene crossover, 
reproduction and natural selection in evolutionary biology [10]. When applying genetic algorithms to a problem, the first step involves representing a chromosome to describe the individuals in the population and encoded them as commonly binary or floating point. The GA then evaluates the 'fitness' of the offsprings within the existing generation. While, the fitness function is dependent the problem being solved [11]. The GA applies the mutation, crossover and selection operations to the individuals in the population to explore all promising regions in the solution space till achieve the needed solution [12].

This advantage of the GA can be applied to neural networks to optimize the topology and/or weight parameters. For a GAbased algorithm employed in fuzzy neural networks, the initial network structure is usually generated or given firstly. The GA is then used to optimize the network's topology in terms of membership functions and/or fuzzy rules [13]. A GA-based algorithm thus has the potential to evolve the number of fuzzy rules, and may be developed as a GA-based pruning method for fuzzy neural networks. The GA is used to determine all the parameters of the membership functions of the fuzzy controller.

\section{PROPOSED GENETIC BASED NEURO- FUZZY CONTROLLER SYSTEM AND ITS APPLICABILITY FOR THE MOBILE ROBOT}

The use of conventional approaches for control problems requires an appropriate model that describes dynamic behavior of the plant to be controlled. However, models are difficult to obtain due to the complexity and nonlinearity of the controlled plant. In order to overcome this problem, other control design approaches which are not relied on nominal models are introduced. One of the successful methods used to solve these problems is the Neuro fuzzy models.

The proposed system is a member in the family of the neurofuzzy systems. It can integrate the genetic algorithm with neuro-fuzzy methodology to optimize their parameters. It has been applied for controlling a mobile robot to manage its operation in a dynamic unstructured environment. It can enable the mobile robot to avoid the obstacles of its path from the start point to its target by intelligently adapting its path.

The suggested a Neuro-Fuzzy controller trained by genetic algorithm. It has the sensors' values of the robot by the fuzzy variable parameters as the reference input, all initialized to zero. The sensor readings from the robot are the inputs to the fuzzy controller and the neural network. The outputs of the fuzzy controller are the speed values for the motors of the robot.

Wherever, the proposed control system uses three sensor input variables representing relative distance between the robot and any object in its environment. These include: Left Distance (LD), Right Distance (RD), and Front Distance (FD). The input variables from the robots' sensors are considered: Not Detected (ND), and Detected (D). While, z-shaped membership function type is defined for the ND grade and s-shaped membership function type for the $\mathrm{D}$ membership function. These membership function types are the variant of Gaussian membership functions that have been proved experimentally by Obe and Dumitrache to be of better performance for the same robot navigation problems [14].

On the other hand, the output fuzzy variables are the motor speed of the robot's wheels, represented by Left Velocity (LV), Right Velocity (RV) and angle $(\theta)$. For the output fuzzy variables, we use three linguistic variables (S-Slow, Z-Zero, and F-Fast) for each of the fuzzy output variables. Proposed system uses Gaussian membership function type for each of the linguistic variables.

The inputs are the sensor readings from the 3- fuzzy input variables ( $\mathrm{LD}, \mathrm{FD}$, and $\mathrm{RD}$ ) of the robot besides history of the last three sensors readings are considered the context readings as the input to our NN. This allows the robot to keep history of the last three previous inputs values from the sensors thereby increasing accuracy of decision of eliciting obstacle avoidance bahaviour.

However, the input of the proposed $\mathrm{NN}$ is the sensor values from the robot and the output is the 6-optimized rule weights to optimize fuzzy logic controller performance for avoiding the obstacles and wall behaviours. The operation of the proposed system can be summarized as follows:

1- The input sensor signal based on the navigational logic for the fuzzy logic trainer was loosely based on the potential fields based method of navigation. The potential fields' method assumed that both the robot and all nearby obstacles possess an electrostatic charge. The negative gradient of the resulting electrostatic field represents the direction that the robot should move. Similarly, the fuzzy logic trainer derived forces that were used to characterize the environment. So, the input to the fuzzy logic trainer was the data collected by the sensors, which resulted in a control strategy that was suitable for training a neural network.

2- The collected data provided a set of 120 data points representing the distance at which an obstacle was detected over its $180^{\circ}$ field of view at about $0.30^{\circ}$ angular intervals. A minimum range of about $10 \mathrm{~cm}$ was selected to and a maximum range of about $50 \mathrm{~cm}$ was selected to ensure that the robot was too far away about the obstacles.

3- The set of force values were sent to both the fuzzy logic trainer and to the neural network as input. The force values were normalized to improve the efficiency of the neural network learning process.

4- The fuzzy logic trainer calculated the ideal steering angle and speed based on the input. This output was used to train the neural network.

5- The outputs from both the neural network and the fuzzy logic trainer were used as inputs to the error backpropagation algorithm. The weights of the neural network were modified to minimize the calculated error.

6- The final corrected output from the neural network was then used to control the vehicle. This was done to ensure that the training of the neural network would be relative to the performance of the neural network itself rather than be dependent on the fuzzy logic navigation

7- The learning algorithm of the neural network in our proposed system is the back propagation. It changes the neural network's weight

8- Genetic algorithm can impact the selection of the optimal number of neurons in the hidden layer of the neural network: (1) The hidden layer must be large enough to approximate the fuzzy logic trainer with sufficient accuracy. (2) The computational burden of the neural network must not minimize its practicality during training or autonomous driving. All the tested neural network sizes 
met this criterion. (3) The initial error during the training process must be low enough to allow the neural network to navigate the unknown obstacles.

While, the structure of the proposed genetic algorithm can be illustrated as:

1. Chromosome Representation: The chromosomes represented as 50 random weight matrix.

2. Initial Population: The initial population for the proposed system constructed from a group of chromosomes generated randomly. The population size is: 30 .

3. Fitness Evaluation: The fitness function used by the system to evaluate the performance of the new individual is minimizing the error value that represents the difference between the actual output of the neural network and the desired one.

4. Selection Methodology: The proposed system uses the ranking selection methodology. The individuals in the population are sorted from best to worst according to their fitness values. Each individual in the population is assigned a numerical rank based on fitness, and selection is based on this ranking rather than differences in fitness.

5. Crossover: Proposed GA uses a single point crossover. Its crossover probability is: 0.65 .

6. Mutation: Single point mutation has a rate is: 0.02 is used in the proposed system

7. Termination: The maximum generations of GA are: 150 .

The proposed system is an intelligent neuro-fuzzy controller system. It can use genetic algorithm to optimize the parameters of the neural network. It has been applied for mobile robot that can move in unstructured danger sites. Table (1) shows a comparison between the minimum training error for various numbers of neurons, along with the resulting correct navigation rate for training and testing. While, table (2), and figures (1,2) illustrates comparisons between the obtained results of the proposed system for those obtained from a traditional neurofuzzy system [15], and neural network system when applied for four different unstructured environments [5].

Table 1: Training error and correct navigation rate (CNR) for training and testing as a function of the number of neurons $(\mathbf{N})$.

\begin{tabular}{|c|c|c|c|c|c|c|}
\hline \multirow{2}{*}{ N } & \multicolumn{2}{|c|}{ Training Error } & \multicolumn{2}{c|}{ Train CNR (\%) } & \multicolumn{2}{c|}{ Test CNR (\%) } \\
\cline { 2 - 7 } & Best & Mean & Best & Mean & Best & Mean \\
\hline 14 & 0.85 & 0.88 & 76 & 72 & 66 & 58 \\
\hline 17 & 0.75 & 0.86 & 82 & 77 & 75 & 62 \\
\hline 20 & 0.71 & 0.80 & 85 & 75 & 63 & 52 \\
\hline 23 & 0.74 & 0.83 & 80 & 70 & 63 & 58 \\
\hline
\end{tabular}

Table 2: Training error and correct navigate of obstacles rate (CCR) for training and testing for alternative unstructured environments.

\begin{tabular}{|c|c|c|c|c|c|c|}
\hline $\mathrm{p}$ & \multicolumn{2}{|c|}{ Proposed System } & \multicolumn{2}{c|}{$\begin{array}{c}\text { Neuro-fuzzy } \\
\text { System 1 }\end{array}$} & \multicolumn{2}{c|}{$\begin{array}{c}\text { Neural Network } \\
\text { System }\end{array}$} \\
\cline { 2 - 7 } & accuracy & $\begin{array}{c}\text { Time } \\
\text { (min.) }\end{array}$ & accuracy & $\begin{array}{c}\text { Time } \\
\text { (min.) }\end{array}$ & accuracy & $\begin{array}{c}\text { Time } \\
\text { (min.) }\end{array}$ \\
\hline Env. 1 & 0.95 & 2.5 & 0.86 & 3.8 & 0.75 & 6.5 \\
\hline Env. 2 & 0.92 & 2.8 & 0.83 & 4.1 & 0.73 & 5.8 \\
\hline Env. 3 & 0.91 & 2.7 & 0.79 & 3.6 & 0.69 & 5.2 \\
\hline Env. 4 & 0.88 & 3.3 & 0.76 & 5.8 & 0.63 & 4.5 \\
\hline
\end{tabular}

From the results of the comparison between the proposed system and the other two algorithms, we find that the proposed system can improve the performance of the neuro-fuzzy controller system by optimize their parameter. Also, the proposed system proves its efficiency when compared with the two other traditional neuro-fuzzy and neural network controller systems. The proposed system can increase the accuracy of the control system and decrease the required time for this operation.

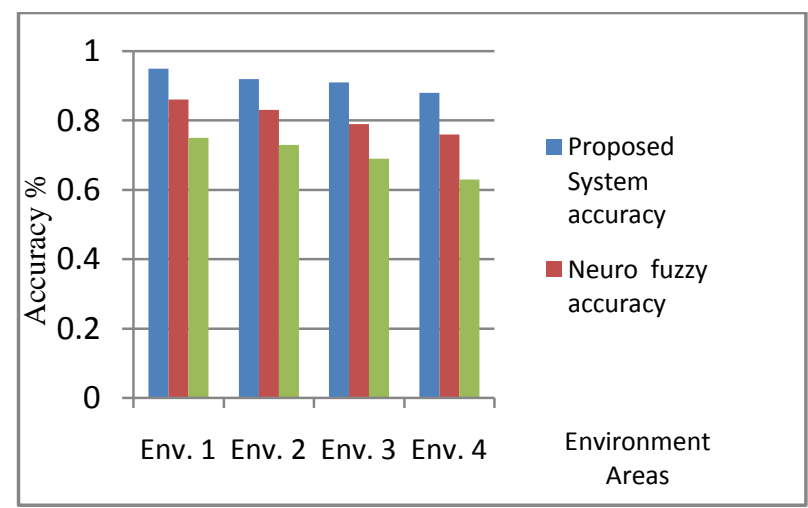

Fig. (1): Comparison of the accuracy of the proposed system and the other system for different environments.

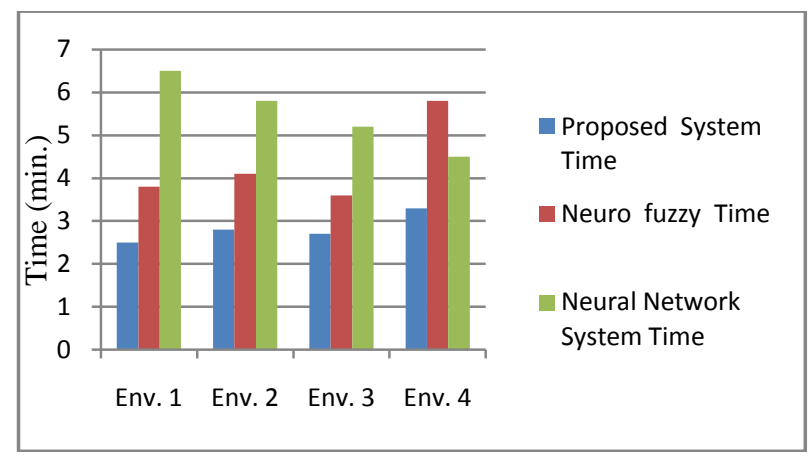

Fig. (2): Comparison of the run-time for the proposed system and the other system for different environments.

For example, when the three systems has run for moving the robot in the Env.1. The proposed system has recoreded an average accuracy $=0.95$ in a time $2.5 \mathrm{~min}$. While, the neurofuzzy system can record an accuracy $=0.86$ in a time $3.8 \mathrm{~min}$. when has run for the same Env.1. On the other hand, the neural network system can has an accuracy $=0.75$ only and at higher time $=6.5 \mathrm{~min}$. However, the proposed system can increase the 
performance of control system rather than the two other control systems.

\section{CONCLUSIONS}

In this research, the proposed neuro-fuzzy controller system introduces optimize of the parameters of the system using the genetic algorithm. It has been applied for a mobile robot to move in unstructured and dynamic environment. The proposed system enables the controller of the mobile robot to adapt its path to avoid the obstacles.

The computer simulation for four types of unstructured environments are carried out to evaluate the performance of the proposed system. The four types of environments that have number of obstacles. Besides, the obtained results from the proposed system are compared with those got from traditional neuro-fuzzy and neural network systems. All the three control systems are run for the same four environments with the same structure and number of obstacles along the robot's path. Hence, the performance of the three control system of avoiding the obstacles is determined. It is found that, the proposed system can achieve higher accuracy in avoiding the obstacles rather than the two other control systems. Also, it has a significant improvement for the time required to complete the path of the mobile robot. Therefore, the proposed system can be used by the mobile robot systems in real and practical situation successfully.

\section{REFERENCES}

[1] I. Dumitrache and M. Dragocea, "Some Problems of Advanced Mobile Robot Control, Extraction Of Fuzzy Rules Using A Self-Organising Fuzzy Neural Network", Fuzzy Sets System, (2006), Vol.150, No.2, pp. 211-243.

[2] D. Nauck, "Neuro-fuzzy systems: Review and prospects". Proc. $5^{\text {th }}$ Euro. Congr. Intelligent Techniques and Soft Computing (EUFIT'97), (1997), pp. 1044-1053.

[3] A. Keles, A. Keles and U. Yavuz, "Expert System Based on Neuro-Fuzzy Rules for Diagnosis Breast Cancer, Expert Systems with Applications, (2011), Vol. 38, No. 5, pp. 5719-5726.

[4] S. Bouzaida and A. Sakly, "Online Control of Nonlinear Systems using Neuro-Fuzzy Design tuned with Cooperative Particle Sub-Swarms Optimization", Proceedings of International Conference on Control, Engineering \& Information Technology (CEIT'13), (2013), Vol.4, pp. 6-10.

[5] S. Wu, M. J. Er and Y. Gao, "A Fast Approach for Automatic Generation of Fuzzy Rules by Generalized Dynamic Fuzzy Neural Networks". IEEE Trans. Fuzzy System, (2001), Vol. 9, No.4, pp. 578-584.
[6] R. Stathacopoulou, G.D. Magoulas, M. Grigoriadou and M. Samarakou, (2011), "Neural-Fuzzy Knowledge Processing in Intelligent Learning Interactive NeuroFuzzy Expert System for Diagnosis of Leukemiam Global", Journals Inc. pp.112-130.

[7] S. Kar, S. Das, P.K. Ghosh, (2014), "Applications of Neuro Fuzzy Systems: A Brief Review and Future Outline", Applied Soft Computing, Vol.15, pp. 243-259.

[8] O.O. Obe and I. Dumitrache, (2012), "Adaptive NeuroFuzzy Controler With Genetic Training For Mobile Robot Control", Int. J. of Computers, Communications \& Control, Vol.6, No.1, pp.135-146.

[9] A.A. Ammar, K. Vamaraju, P. Mukherjee and J. Gorchynski, (2011), "Optimized Fuzzy Logic Training of Neural Networks for Autonomous Robotics Applications", International Journal of Scientific \& Engineering Research. 2(10):201-214.

[10] Jang, J. ; Sun, C. and Mizutami, E. (1997), "Neuro-Fuzzy and Soft Computing: A Computational Approach to Learning and Machine Intelligence". New Jersey: Prentice Hall, First ed., pp.123-153.

[11] L. Cristaldi, M. Lazzaroni, A. Monti, and F. Ponci, (2004), "A Genetic Algorithm For Fault Identification in Electrical Drives: A Comparison With Neuro-Fuzzy Computation", Proceedings of the Instrumentation and Measurement Technology Conference (IMTC 04), Vol. 2, pp. 1454-1459.

[12] T. Tettey, and T. Marwala, (2006), "Controlling Interstate Conflict using Neuro-fuzzy Modeling and Genetic Algorithms", INES 2006 - 10th International Conference on Intelligent Engineering Systems, pp. 1210-1218.

[13] G. Leng, T.M. Martin, and G. Prasad, (2006), "Design for Self-Organizing Fuzzy Neural Networks Based on Genetic Algorithms", IEEE Transactions on Fuzzy Systems, Vol.14, No.6, pp.755-765.

[14] O.O. Obe, and I. Dumitrache, (2010), "Fuzzy Control of Autonomous Mobile Robot", U.P.B. Sci. Bull., Series C, Vol. 72, No. 3, pp. 150-162.

[15] G.Leng, G. Prasad and T.M. McGinnity, (2004), "An OnLine Algorithm for Creating Self-Organizing Fuzzy Neural Networks", Neural Networks, Vol.17, pp. 1477 1483 .

Amira H. Mohamed, Associated Prof. of Computer Engineering, National Centre for Radiation Research and Technology. 